\title{
I sonetti dell'esperienza. Montale traduttore di Shakespeare
}

\author{
Francesco Erspamer
}

1.1. Composte negli anni antecedenti al 1938 e pubblicate solo a partire dal 1944, le versioni di tre sonetti di Shakespeare - il 22, il 33 e il 48appartengono senz'altro alla straordinaria tarda fase della seconda stagione montaliana, per intenderci il periodo dei Mottetti, della IV sezione delle Occasioni (la quale infatti reca in epigrafe due versi dei Sonnets), e anche di Finisterre e delle Silvae. Una produzione che è espressione di un momento di crisi, paura e sconforto prima, guerra e desolazione poi; nulla di strano che Montale proprio allora si accostasse - oltre che, per motivi in parte coincidenti, ai romanzieri americani-a Shakespeare, alle sue opere più alte e più inquiete, il Sogno di una notte di mezza estate, l'Amleto, il canzoniere. A Shakespeare, cioè a un colosso della letteratura, dunque un punto fermo fra tante incertezze, ma a Shakespeare anche in quanto uomo e artista in un'altra età di dubbi e sconvolgimenti. Non a caso pure negli anni seguenti, finita la carneficina ma non l'orrore ("La purga dura da sempre"), ancora il teatro elisabettiano (si ricordino le versioni della Commedia degli errori, del Timone di Atene, del Giulio Cesare, del Racconto d'inverno, del Faust di Marlowe) costituì il terreno in cui preferibilmente amò scavare il traduttor Montale. ${ }^{1}$

Occorre tuttavia distinguere fra le opere drammatiche e le composizioni liriche. Le prime sono più che altro il frutto di quella che il poeta stesso ebbe a definire la sua "forzata e sgradita attività di traduttore" (Sulla poesia 567), cioè del lavoro che fu costretto a svolgere, in parte per esigenze economiche, nel rispetto dei rigidi criteri "di quasi letteralità e di assoluto scrupolo filologico" (Amleto 207) imposti dagli editori e dalle collane per le quali erano commissionate. Al contrario i rifacimenti delle poesie (non solo quelle di Shakespeare) sembrano un prodotto più spontaneo, e comunque più congeniale alla sua arte: non solo per la ragione quasi ovvia che egli si ritrovava meglio nella misura più breve, ma anche perché ben diverse possibilità di intervento gli erano concesse. Che poi sia proprio da questa franchigia che nascano, sempre, le migliori traduzioni, che solo attraverso il paradosso di una nuova scrittura del già scritto si riesca a salvare la poesia, a trasmetterla attraverso la barriera della trasformazione linguistica, è un problema metodologico che qui ci riguarda solo marginalmente; il punto importante è che proprio in questa direzione si sia mosso Montale non appena fu libero di leggere $\mathrm{e}$ interpretare da poeta i suoi autori. 
Ogni traduzione veramente riuscita, sostiene Walter Benjamin, "tocca l'originale di sfuggita e solo nel punto infinitamente piccolo del senso, per continuare, secondo la legge della fedeltà, nella libertà del movimento linguistico, la sua propria vita" (48). Montale questa "legge della fedeltà" non la rispetta, e il "punto del senso" non lo tocca, lo sfiora soltanto. La poesia che può nascere è soltanto sua, per una volta germogliata non su un'occasione della vita, non su un sogno o un ricordo, ma su un altro testo, su un palinsesto letterario. I fondamenti teorici di questa scelta di infedeltà li veniva del resto esponendo, proprio in quegli anni, in alcuni saggi. Intorno al 1942 scrisse che "la poesia, assai più delle altre arti, sembra soggetta a invecchiare," destino cui riesce a scampare solo se "si presta ad essere ricostruita e interpretata in modo diverso, a essere fonte di altissimi equivoci" (Sulla poesia 103). Quattro anni dopo, nell'"Intervista immaginaria," confermò i legami che sussistevano fra il suo mestiere di poeta e quello di traduttore, ricordando quanto il secondo avesse pesato nella sua "lotta per scavare un'altra dimensione nel nostro pesante linguaggio polisillabico" (Sulla poesia 567), insomma nella creazione di una poesia che, come ha scritto recentemente Ghanshyam Singh, "sembrava inglese, anche se scritta in italiano, e anche se nessun poeta inglese aveva mai scritto una cosa simile" (207). Il testo a fronte, nelle prime edizioni del Quaderno di traduzioni come qui sotto, è perciò soltanto un altro grimaldello per entrare, ancora una volta, nell'officina poetica di Montale, un pretesto per scoprirne i meccanismi di "ri-uso," di "récriture," e forse anche per meglio comprendere il cammino della sua lingua dagli Ossi alla Bufera. Solo le traduzioni, espunta ogni "filigrana di originale straniero,"2 possono dunque entrare nel canzoniere, nel Libro in senso stretto, ma vi entrano a pieno titolo: ed è come opere assolutamente autonome e interamente montaliane che mi propongo qui di analizzarle.

1.2. Non è una novità che inglese e italiano posseggano due diverse 'velocità'; che, come notò un altro straordinario interprete di Shakespeare, Giuseppe Ungaretti, "in un medesimo gruppo di vocaboli la quantità di sillabe italiane sia superiore alle inglesi nel rapporto di circa sedici su dieci o undici" (13). Ciò comporta dei problemi in fase di traduzione; i Sonnets, ad esempio, sarà impossibile riuscire a renderli nella nostra lingua senza rinunciare a qualcuna delle loro caratteristiche: la rima, la struttura metrica, quella fonica e melodica, la relazione fra le unità di senso e le unità prosodiche. Di fronte all'alternativa, Montale, unico fra i traduttori recenti, preferì rispettare la metrica e accettare la semplificazione del contenuto. Come ha scritto Gilberto Lonardi, egli amava "cercarsi una forma già data con cui fare i conti" (153). Tuttavia la costanza con cui, in tutti e tre i rifacimenti, mantenne le misure dell'endecasillabo (con un'alternanza di a maiore e di $a$ minore e una grande mobilità degli accenti secondari e delle cesure) e del sonetto elisabettiano (tre quartine a rime alterne più un distico a rima baciata: $\mathrm{ABAB} / \mathrm{CDCD} / \mathrm{EFEF} / \mathrm{GG}$ ), si rivela infine, più che la dimostrazione di una 
volontà di rispettare le rigide forme shakespeariane, un ennesimo esempio della sua ben nota "ironia metrica." Si noti l'uso frequente della rima imperfetta, anche quando sarebbe stato agevole trovare quella esatta; oppure la grande quantità di enjambements; o ancora il sapiente gioco di rime al mezzo, naturalmente complicato da equivocità. In un solo caso troviamo la rima mascherata entro la parola sdrucciola ("Son. XXII": "riprendere"-"rende"), ma spesso assonanze e consonanze legano fra loro le coppie di rime. Ad essere confermata è insomma "la singolare ambiguità del metro montaliano, che può essere letto sia seguendo gli a capo indicati nella pagina, sia capovolgendo la struttura secondo i suggerimenti delle rime al mezzo" (Barberi Squarotti 202); ambiguità presente anche nell'ossatura ritmica a causa della polivalenza delle rime. Né questa azione di svuotamento delle strutture metriche resta un divertissement fine a se stesso; al contrario essa è il preciso segnale - lo vedremo-di un analogo processo di corrosione al livello del significato.

2.1. Il primo sonetto che intendo esaminare è il XXXIII. Eccone il testo, immediatamente seguito dall'originale shakespeariano:

Spesso, a lusingar vettc, vidi splendere sovranamente l'occhio del mattino, e baciar d'oro verdi prati, accenderc pallidi rivi d'alchimic divinc.

Poi vili fumi alzarsi, intorbidata d'un tratto quella celestiale fronte, e fuggendo a occidente il desolato mondo, l'astro cclare il viso e l'onta. Anch'io sul far del giorno ebbi il mio sole e il suo trionfo mi brillò sul ciglio: ma, ahimè, poté restarvi un'ora sola, rapito dalle nubi in cui s'impiglia.

Pur non ne ho sdegno: benc può un terrestre sole abbuiarsi, se è così il celeste.

Full many a glorious morning have I seen flatter the mountain-tops with sovereign cye, kissing with golden face the meadows green, gilding palc streams with heavenly alchemy; anon permit the basest clouds to ride with ugly rack on his celcstial facc, and from the forlorn world his visage hide, stealing unsecn to west with this disgrace: cven so my sun one carly morn did shine with all triumphant splendour on my brow; but out, alack! he was but onc hour minc, the region cloud hath masked him from me now.

Yet him for this my love no whit disdaincth; suns of the world may stain when heaven's sun staineth. 
Il testo di Shakespeare, come appare fin da un primo sguardo, è di estrema semplicità sul piano tematico: a una descrizione naturalistica del sorgere del sole, del suo oscurarsi dietro le nuvole e del suo avviarsi al tramonto, segue lo scioglimento della metafora attraverso l'indicazione dell'occasione biografica che l'ha generata. Una sentenza apodittica conclude il componimento. Ma sopra questa sottile trama narrativa si innesta, e la poesia vive grazie a esso, un preciso e complesso rinvio ad altri codici, un intenso ricorso a stratificate connotazioni linguistiche e culturali. A questo livello avviene innanzi tutto l'antropomorfizzazione del cosmo, la quale a sua volta comporta l'identificazione di "sun" (omofono di "son" "figlio') con il re (riconoscibile dai tradizionali attributi: "glorious," "sovereign," "golden," "celestial") e la conseguente utilizzazione del particolare linguaggio della corte elisabettiana. Ma un secondo registro si sovrappone: quello dell'alchimia (pratica, allora, di notevole peso sociale), di nuovo una metafora nella metafora con l'aggancio della figura dell'alchimista al sole e della sua arte alla luce (cfr. Serpieri 125-38).

La traduzione di Montale appare, a una prima lettura, praticamente perfetta, capace di conservare dell'originale non solo il senso letterale, ma pure i principali sensi allegorici. L'identificazione del sole con il sovrano è suggerita da numerosi termini polisensi, distribuibili in due serie: 1) "occhio," "baciar," "pallidi," "alzarsi," "fronte," "fuggendo," "viso" (antropomorfizzazione del cosmo); 2) "lusingar," "sovranamente," "oro," "celestiale" (specificazione della dignità regale). E persino i rifcrimenti al registro alchimistico, piuttosto estranei alla lirica novecentesca (ma si ricordi il "piombo fuso a mezzanotte" di "Carnevale di Gerti"), sono mantenuti: il centro è ovviamente "alchimì," ma si collocano in quell'area semantica anche "baciar d'oro," "accendere," "vili" (fa pensare ai 'metalli vili,' opposti dell'oro, esattamente come in inglese "basest" rimanda ai 'base metals'), "fumi," "intorbidata." Alcuni elementi, come si vede, sono pluri-isotopi.

La maggior parte delle variazioni rispetto al testo shakespeariano può essere, in questa prospettiva, imputata alla diversità degli strumenti linguistici, oppure alla peculiarità del modo di poetare montaliano. Così, in presenza di ripetizioni di vocaboli, Montale preferì modificare la struttura della frase 0 , dove fosse possibile, utilizzare dei sinonimi. Così la coppia "clouds"-"cloud" è resa con "fumi"-"nubi"; "morning"-“morn" con "mattino"-"far del giorno"; "world"-"of the world" con "mondo"-"terrestre"; "heavenly"-"heaven's" con "divine"-"celeste" (ma in questo caso si provoca la ripetizione, assente in inglese, "celestiale"-“celeste"); "seen"-"unseen" con un singolo "vidi."

Straordinario è poi il modo in cui la ricchezza omofonica del sonnet è stata riprodotta in italiano. La massiccia presenza (spesso in rima) del suono /ai/, riflesso del pronome personale "I" collocato in posizione di evidenza nel primo verso ("I," "eye," "alchemy,,"3 "ride," "hide," "my," "shine," "triumphant," "mine," "my"), trova riscontro nel ricorrere, subito dopo la comparsa del solitario "io" (altrove il pronome è sempre sottinteso), del dittongo/io/ 
(nelle prime due quartine ritrovabile soltanto in "occhlO," e non più ripetuto nei quattro versi conclusivi):

Anch'lo sul far del glOrno cbbi il mIO sole c il suo trlOnfo mi brillò sul ciglıO.

E l'affollamento di sibilanti è in entrambi i couplets:

Yet him for thiS my love no whit diSdaineth;

SunS of the world may Stain when heaven'S Sun Staineth.

Pur non ne ho Sdegno: bene può un terreStre

Sole abbuiarSi, Sc c̀ coSì il celcStc.

Da un punto di vista melodico, inoltre, sia Shakespeare che Montale riescono a far corrispondere ai due momenti tematici, la metafora del sole e il suo scioglimento, due diversi ritmi: nei primi otto versi prevalgono i vocaboli lunghi e poco cadenzati (per es., "e fuggendo a occidente il desolato"), nei restanti sei, al contrario, le parole corte e dagli accenti marcati (in italiano visibili anche graficamente: "brillò," “ahimè," "poté," "può," “è," "così").

2.2. Al lettore puntiglioso tuttavia non sarà sfuggito che nei vv. 11-14 il "Sonetto XXXIII" nettamente si allontana dal testo inglese. $\dot{E}$ il primo segnale dell" "infedeltà" montaliana. Si può sospettare che il graduale impoverimento degli ultimi versi shakespeariani, la chiusura in tono minore - accettazione pessimistica dell'imperfezione, che finisce con lo sgretolare anche il linguaggio che la veicola, - abbiano spinto Montale a una maggiore autonomia d'ispirazione, che gli concedesse di trasformare quel movimento discendente in una fase di preparazione al climax conclusivo, al distico in cui far esplodere la sentenza epigrammatica. Ma ciò che realmente conta è l'effetto di questi mutamenti, la loro funzione. Essi modificano il senso di tutta la poesia, che solo alla fine - o meglio, a una seconda lettura-diventa correttamente interpretabile nel suo più autentico significato.

Per dimostrare l'autonomia semantica, oltre che linguistica, del "Sonetto," è necessario esaminarne più da vicino il lessico. Indubbiamente esso appartiene a Montale, in particolare al Montale degli anni '30 e '40. Solo pochissimi termini non ricordo di averli incontrati nelle ultime Occasioni o nei più antichi componimenti della Bufera. La gran parte ricorre, e fra essi tutti quelli semanticamente più rilevanti (con la sola, prevedibile eccezione di "alchimie"): "occhio," "oro," "fumi," "fronte," "viso," "giorno," "sole," "ciglio," "nubi," per i quali proprio non serve portare esempi; e poi "astro" (almeno "Stanze"; ma già, in Ossi di seppia, "Arsenio"); c gli aggettivi: "verdi" (frequente, in tutte le tonalità; e si ricordino le "isole verdi" di "Keepsake" e l'"anima verde" di "L'anguilla"), "celestiale"-"ccleste" (cfr. "Elegia di Pico Farnese," "Il giglio rosso," "Iride"); e i verbi: "rapito" (cfr. "Gli orecchini," "Nella serra"), "accendere" (cfr. "L'anguilla"), "s impiglia" (cfr. "L'arca"), "abbuiarsi" (cfr. "Dora Markus" II), "cclare" (più volte, ma 
si noti in "L'orto": "là dove acri tendìne / di fuliggine alzandosi su lampi / di officine celavano alla vista / l'opera di Vulcano"), "brillò" (cfr. "Costa San Giorgio," "Nella serra," "L'anguilla"). Congeniale a Montale è poi l'uso di "intorbidata" in riferimento alle nebbie che si alzano lungo i monti: "Le fumate / morbide che risalgono una valle / . . fino al cono diafano / della cima m'intorbidano i vetri" ("Notizie dall'Amiata" I).

Piuttosto evidenti, in particolare, le analogie con "Corrispondenze" (1936): anche lì un'atmosfera fumosa ("un miraggio / di vapori"), alchimistica ("matura incubi d'oro"), anche lì il colore verde ("picchio verde"), anche lì vette e acque illuminate ("toppe arse dei colli"; "oro / a specchio delle gore"), anche lì, infine, una conclusione all'insegna dell'occultamento dietro densi vapori ("alla febbre nascosta dei diretti / nella costa che fuma").

2.3. Come già la composizione shakespeariana, e forse in misura persino maggiore, il "Sonetto XXXIII" presenta una perfetta organizzazione interna. La prima quartina celebra il trionfo del sole (indicato solo metaforicamente: "occhio del mattino"), la seconda quello delle nubi (anch'esse non nominate che per traslato: "fumi"); la terza riproduce in spazio dimezzato i due momen$\mathrm{ti}$, riconducendoli a una dimensione non più generale, cosmica, bensì privata: vv. 9-10, epifania del sole (questa volta esplicitamente: "sole"), vv. 1112: epifania delle nuvole (esplicitamente: "nubi"). La struttura retorica può dunque essere rappresentata nel seguente modo:

\section{EPIFANIA DEL SOLE}

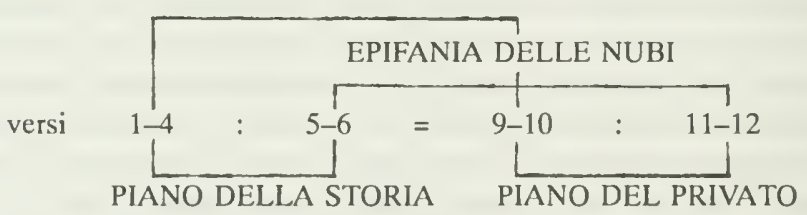

Il distico conferma l'equazione: l'ossimoro "sole abbuiarsi" (ripreso nel Quaderno di quattro anni, "L'educazione intellettuale": "un luminoso buio") recupera il motivo della lotta fra luce e oscurità; l'opposizione (in rima) "celeste" vs "terrestre" rende il binomio Storia vs Individuo (oltre che, ovviamente, quello Uomo vs Dio: cfr. "L'angelo nero": "non celestiale né umano"):

terrestre / sole abbuiarsi, se è così il celeste.

Entrambi i motivi, il tema della sfiducia nella storia e nell'individuo, e l'immagine del sole offuscato dalle tenebre, sono di fondamentale importanza nella poesia di Montale. Vediamo di esaminarli più in dettaglio.

La dicotomia fra dimensione pubblica e dimensione privata è sancita, oltre che sul piano ritmico, su quello sintattico. Nelle prime due quartine l'uso di forme verbali non personali ("lusingar," "splendere," "baciar," "accendere," "alzarsi," "intorbidate," "fuggendo," "celare") crea un effetto di indetermina- 
tezza e di universalità, accresciuto dalla equivocità grammaticale della seconda strofe, in cui non è immediatamente chiaro se "fronte," "mondo," "astro," "viso" e "onta" siano soggetti o complementi oggetto. Al contrario, e con effetti opposti, i vv. 9-12 sono caratterizzati dalla frequenza dei possessivi e dei sostituti personali ("io," "mio," "suo," "ahimè," "restarvi," "cui," "s 'impiglia”). La ricorrenza del suono /io/ conferma la posizione di preminenza che nella terza quartina spetta al soggetto in prima persona.

Quanto al significato e al valore della storia e dell'esperienza privata in Montale, motivi dominanti in Salura, molto è stato scritto: al già fatto non resta dunque che rinviare. ${ }^{4}$

2.4. Non c'è dubbio che l'aurora rappresenti uno dei temi più caratteristici e ricorrenti dell'imagery montaliana. Gli esempi sarebbero numerosissimi: forse a torto si è privilegiata, una volta individuata nel crepuscolo l'ora topica delle Occasioni e della Bufera, la sera (cfr. Avalle 83-84) a dispetto dell'alba. I due momenti di trapasso non possono invece essere né contrapposti né separati; essi si confondono, si identificano ("un mattino / più lungo era la sera," in "Barche sulla Marna”), e insieme costituiscono un ampia zona temperata, di superfici lucide e appannate, di contorni imprecisi, che si oppone agli eccessi del giorno e della notte, e che si allarga oltre $\mathrm{j}$ consueti confini temporali, venendo spesso il bagliore solare attenuato dalle nebbie, i] buio da lampi e stelle. Proprio a questi fattori di moderazione è assegnato in genere un valore positivo, legandoli a immagini "alte," poetiche: "Una mandria lunare sopraggiunge / poi sui colli, invisibile, e li bruca" ("Bassa marea"); "l'orizzonte di rame / dove strisce di luce si protendono / come aquiloni al cielo che rimbomba / (Nuvole in viaggio, chiari / reami di lassù! D'alti Eldoradi / malchiuse porte!)" ("Corno inglese").

Si ricordi la conclusione di "La primavera hitleriana," senza fraintenderla: "un'alba che domani per tutti / si riaffacci, bianca ma senz'ali / di raccapriccio, ai greti arsi del sud ..." L'aurora non annuncia niente, non è metafora di un inizio, di una nascita o di una rinascita; è essa stessa il punto di arrivo. La sua contraddittorietà, la sua fugacità, sono l'unica meta cui l'uomo può aspirare. E neppure parlerei di "fiducia resistenziale" (Carpi 111), perché non c'è nessuna sicurezza, nessuna certezza: il bene è solo un non-male, il vero un non-falso, come il crepuscolo è un non-giorno e una non-notte. La solarità degli Ossi è perduta ormaj, e l'astro diurno è al massimo un povero, debole sole (ricorda quello di Sergio Corazzini), "freddoloso" ("Ti libero la fronte dai ghiaccioli"), "senza caldo" ("La rana ..."), "grigio" ("Proda di Versilia"), facilmente sconfitto: "al giorno / primo già annotta" ("Personae separatae"); al quale fa pendant uno strano sole notturno, "cieco" ("La primavera hitleriana"), "nero" ("Iride"). A un incerto mattino, o al ripiombare della notte, è allora unica alternativa - ma forse neppure questa scelta ci è data - quell" "alba infinita e senza strade, / dov'è la lunga attesa" ("Barche sulla Marna").

Su un piano strettamente retorico, l'immagine del sole nascente è com- 
plementare, ai fini dell'istituzione e della chiarificazione di una complessa allegoria della conoscenza umana, a quella delle nuvole. Ma anche al livello dei significati le due immagini non sono antitetiche: se alla prima non spetta il ruolo di pars construens del componimento, la seconda non può essere interpretata come pars destruens. Di nuovo, la loro funzione è piuttosto quella di mescolarsi, a realizzare e a conservare una mediocritas non certo aurea ma almeno vivibile: "al chiaro e al buio, soste ancora umane / se tu a intrecciarle col tuo refe insisti" ("Al primo chiaro, quando"); "poca vita tra sbatter d'ombra e luce" ("Sotto la pioggia").

Le nubi e $\mathrm{i}$ vapori sono infatti attributi essenziali del crepuscolo: "È giorno fatto. / A un soffio il pigro fumo trasalisce" ("Perché tardi? Nel pino lo scoiattolo"), "all'alba . . . / il fumo delle mine s'inteneriva, / saliva lento le pendici a piombo" ("Punta del Mesco"), ecc. Come del resto era già stato in uno dei riconosciuti "padri metafisici" di Montale, Baudelaire: "Les maisons ça et là commençaient à fumer," "Un mer de brouillards baipuait les édifices" ("Le crépuscule du matin"). Il loro valore positivo è riscontrabile lungo tutto l'arco della poesia montaliana, fin dal 1923 e dalla "nuvola grandiosa" di uno dei Sarcofaghi, "Ora sia il tuo passo." In Finisterre c'è addirittura l'identificazione della foschia mattutina con la donna amata: "e irrequieta la tua fronte / si confonde con l'alba, la nasconde" ("La frangia dei capelli . ..."); processo metaforico esplicitamente confermato nell" "Intervista immaginaria": "Ho proiettato la Selvaggia o la Mandetta o la Delia dei Mottetti sullo sfondo di una guerra cosmica e terrestre, senza scopo e senza ragione, e mi sono affidato a lei, donna o nube, angelo o procellaria" (Sulla poesia 568). Ancora in Satura la nebbia è un elemento caratteristico del "visiting angel": "o angelo nero ... / non dissipare la nebbia che ti aureola" ("L'angelo nero");" ma la chiave di lettura del topos va cercata parecchio a monte, in una delle grandi elegie riassuntive di Ossi di seppia, "Fine dell'infanzia":

Un'alba dové sorgere che un rigo

di luce su la soglia

forbita ci annunziava come un'acqua;

e noi certo corremmo

ad aprire la porta

stridula sulla ghiaia del giardino.

L'inganno ci fu palese.

Pesanti nubi sul torbato mare

che ci bolliva in faccia, tosto apparvero.

Era in aria l'attesa

di un procelloso evento.

Strania anch'essa la plaga

dell'infanzia che esplora

un segnato cortile come un mondo!

Giungeva anche per noi l'ora che indaga.

La fanciullezza era morta in un giro a tondo. 
L'ora delle nubi, dell'alba che dové sorgere, è "l'ora che indaga," come in "Falsetto": "Esterina, i vent"anni ti minacciano, / grigiorosea nube / che a poco a poco in sć ti chiudc."

2.5. Alla luce di questo scavo "archeologico" le nubi e i fumi che offuscano il sole nel "Sonetto XXXIII" finalmente si rivelano una allegoria dell'esperienza, della maturità, che smaschera suo malgrado le fascinose ma fallaci illusioni dell'infanzia e dell'ignoranza. Esiti pienamente montaliani, questi, che si ripercuotono, modificandone il senso, sui motivi ereditati dal testo inglese: l'alchimia, per esempio, diventa semplice traslato della finzione, dell'inganno (lusinga, spaccia per oro ciò che è "pallido"). E nclle ultime strofe non si verifica lo sgretolamento del linguaggio, perché non c'è fallimento, ma lucida e necessaria conquista di un'amara verità.

Più da vicino. Che funzione salvifica sia quella delle nubi, e che comunque queste non siano connotate negativamente, è rilevabile sul piano della sintassi e del lessico. Ecco Montale dimenticare quell" "hath masked" di cui Shakespeare faceva soggetto "the region cloud": perché le sue nuvole non nascondono, non mentono, ma al contrario svelano. E sostituirlo con "s'impiglia": un rapimento, si, ma un rapimento di cui si fa complice lo stesso oggetto "rapito." Più sopra il duro "ugly ('turpe') rack" ('cumulo-nembo,' ma anche 'strumento di tortura') si dissolve in un "intorbidata" di cui può essere soggetto "fumi" ma anche, con costrutto assoluto, "fronte." Eे di "astro," inoltre, che sono predicati i termini meno nobili: "fuggendo," "celare," "onta." Sembra che l'ufficio dei "fumi" ("vili" in quanto troppo facilmente vittoriosi su un avversario inadeguato, troppo fragile; dunque 'pusillanimi,' non 'spregevoli' o 'abietti') sia solo quello di incutere timore: il sole si oscura da sé, o si impiglia, al semplice apparire di chi lo possa (s)mascherare.

Infatti il sole, mi sembra scnza dubbio, rappresenta le illusioni dell'uomo. Prima chimera, la natura: le "vette," i "verdi prati," i "pallidi rivi," cioè il "sole (= paradiso) terrestre"; immagini idilliche, da "poeti laureati." Fanno presto luogo a quel dolorosissimo, spezzato (anche graficamente, nei due versi) "desolato / mondo." Seconda chimera, la vita, il sogno della giovinezza che si crede eterna: quel "far del giorno" che pare promettere luminosi sviluppi. E invece "un'ora sola" è tutto ciò che ci spetta. Infine, la grande illusione di Dio: è a Lui (e non a un re terreno, come in Shakespeare) che alludono gli attributi, necessariamente antropomorfi ("la nostra debole mente non può fare a meno di raffigurarsclo come Persona" scriverà Montale stesso in Auto da fé 350), disseminati nelle prime due quartine. È Lui l" "occhio" che splende "sovranamente" nel mattino, effigie mutuata da una ben attestata tradizionc figurativa; è Lui il sole "celeste" (si ricordi "Nella serra": "l"oscuro / pensiero di Dio discendeva / . . . tra suoni / celesti"; c le "tessitrici celesti" di "Tempi di Bellosguardo" III). Una illusione, appunto: denunciata già a livello testuale dall'uso di "astro," cioè di un termine ormai fortemente connotato nel senso di una pscudo-scienza della divinazione. l'astrologia: e 
soprattutto dal fatto che a essere definite "divine" siano le "alchimie" (naturalmente l'aggettivo non può non rimandare, nella doppia direzione delle fonti e degli esiti, alla "divina Indifferenza" degli Ossi, "Spesso il male di vivere ho incontrato," e alla "divina inesistenza" del Quaderno di quattro artni, "Domande senza risposta").

Tutte parvenze, dunque, che all'apparir del vero si dichiarano fatue; e che pure mantengono un certo valore positivo. I miraggi, le utopie, persino le menzogne, non sono inutili: esse sono comunque dei "significati," sono le briciole di speranza di cui vive l'uomo. "Quando dico che probabilmente il mondo non esiste mi guardo bene dal pretendere che questa inesistenza sia priva di un significato positivo: ha certo un significato il fatto che il mondo, per noi, esista" (Intervista 27).

3.1. Dei tre, il "Sonetto XXII" è l'unico che presenti varianti di un certo rilievo; ne propongo pertanto le principali di seguito alla redazione definitiva $($ Cit = Città 1944; Uo = Uomo 1945):

Allo specchio, ancor giovane mi credo ché Giovinezza e te sictc una cosa.

Ma se una ruga sul tuo volto io veda saprò che anche per me morte non posa. Quella beltà che ti ravvolge è ancora parvenza del mio cuore che nel tuo alberga - e il tuo nel mio-; e come allora deciderc chi è il vecchio di noi due?

Poni in serbo il tuo cuore, ed io lo stesso farò di me: del tuo così zelante come fida nutrice in veglia presso la cuna, che ogni morbo stia distante.

Spento il mio cuore, invano il tuo riprendere vorresti: chi l'ha avuto non lo rende.

1-2] Allo specchio, guardandomi, mi credo giovane perché tu sei Giovinezza. (Cit Uo) 4] saprò che anche i mici giorni mortc spezza. (Cit) 12] la culla, che ogni morbo stia distante. (Uo)

My glass shall not persuade me I am old, so long as youth and thou are of onc date; but when in thee time's furrows I behold, then look I death my days should expiate. For all that beauty that doth cover thee is but the seemly raiment of my heart, which in thy breast doth live, as thine in me; how can I then be elder than thou art? O therefore, love, be of thysclf so wary as I, not for myself, but for thee will; bearing thy heart, which I will keep so chary 
as tender nurse her babe from faring ill.

Presume not on thy heart when mine is slatin;

thou gav'st me thine, not to give back again.

Sulla tematica del testo shakespeariano si può questa volta sorvolare frettolosamente: i motivi dello specchio rivelatore e della donna specchio del poeta, della mano del tempo che traccia le rughe e del trasferimento del cuore dell'amante nel petto della persona amata, sono frequenti non solo in Shakespeare (cfr. i sonetti 2, 3, 19, 77, 103), ma in tutta la tradizione del petrarchismo europeo. Da un punto di vista retorico, il somnet è fra i più efficaci del canzoniere.

3.2. La divaricazione dell'interpretazione montaliana rispetto all'originale appare ancora accresciuta. Un confronto dei due incipil ce ne dà subito la misura. Diversa è anzitutto l'età del protagonista; o meglio, diversa è la "qualità" delle informazioni che sull'età del protagonista ci danno i duc testi. Quello inglese mette in risalto la discordanza fra il responso dello specchio, che riflette l'immagine, veritiera, di un vecchio, e il convincimento del personaggio che dice "io," che non se ne lascia persuadere. Nella traduzione, al contrario, lo specchio è un semplice strumento, che fornisce dati da interpretare: i ragguagli non sono pertanto diretti, riferibili a una realtà obiettiva, ma sono nella loro totalità mediati dall'opinabile giudizio del narratore ("ancor giovane mi credo"). Nel prosieguo delle quartine queste differenze avranno significative conseguenze. In Shakespeare la descrizione del destinatario interno (o narratario), del "thou" insomma, conferma le indicazioni dello specchio, e questa volta il narratore si convince ("but when in thee time's furrows I behold, / then ..."): la morale è che il tempo non lo si misura con parametri inerti, sia pure obiettivi, ma dalle tracce che lascia sulle cose e sulle persone care. In Montale, invece, il "volto" dell'amata, in cui il poeta si osserva, restituisce una sembianza diversa da quella resa dallo specchio: e la conclusione è che il tempo non esiste, che neppure la realtà esiste, che tutto è ambiguità, mutevolezza e incertezza.

La ragione di tale divaricazione si rivela peraltro assai più profonda dell’intenzione di eliminare "tutte le immagini, così rapide e varie in inglese, che sembrano attribuire a dei concetti, o a dei meri oggetti, stati e comportamenti umani"; né tali immagini possono venire considerate "un po' incongrue per una fantasia italiana” (Meoli Toulmin 459-60). Si ricordi almeno la personificazione dello "specchio annerito" di “Dora Markus" Il (1939), nell'occasione dotato di orecchie, oltre che di occhi e memoria: "[La sera] dice / allo specchio annerito che ti vide / diversa una storia di errori." II "tradimento" del testo shakespeariano si può piuttosto giustificare, a livello formalc, con il gusto per una bimembrazione più marcata, costruita sulla coppia (isotopa anche grammaticalmente) "specchio"."volto"; ma più importante è che, alla base, c'era un diverso significato da esprimere.

3.3. Passando all'esame del lessico, si è di primo acchito portati a soste- 
nere che $\mathrm{j}$ vocaboli siano soprattutto di retaggio aulico, e che questa scelta sia intenzionalmente operata per creare un alone di antichità, di classicità. In effetti numerosi termini sono obsoleti e letterari: "ché," "posa" (nel senso di 'riposa"), "beltà," "parvenza," "alberga," "fida," "nutrice," "cuna," "morbo"; $\mathrm{e}$ in almeno un caso il calco ritmico e sintattico di un verso dantesco è evidente: "ché Giovinezza e te siete una cosa" deriva dalla Vita nuova, "Amore e 'l cor gentil sono una cosa" (20.3). Tuttavia non direi che questo repêchage sia dettato dal desiderio di mantenere una "fedeltà linguistica ai sonetti shakespeariani, al contesto culturale in cui si situano, e al loro carattere d'esemplarità nella storia letteraria inglese" (Musatti 129-30). Piuttosto va notato che verso la grande poesia trecentesca si era indirizzato il Montale di quegli anni, per sua stessa ammissione: "Le poesie di Finisterre . . rappresentano la mia esperienza, diciamo così, petrarchesca" (Sulla poesia 568). Scriveva giustamente Piero Bigongiari: “I sonetti shakespeariani, lo splendido romanzo che li sottende, non sarebbero stati immaginabili tradotti dal primo Montale; è proprio il Montale petrarchesco di Finisterre che può adoperare il linguaggio lucido e psicologico, di resa sentimentale diretta, di questo Shakespeare" (238).

In effetti, ben prima che dantesco o petrarchesco (o leopardiano), il lessico di questo sonetto è, ovviamente, montaliano. Se su "cuna" (già nella prima redazione; e la forma fu ripristinata dopo l'infelice variante in "culla" di Citıà) inevitabilmente pesa la suggestione di Leopardi e della chiusa del "Canto notturno" ("dentro covile o cuna, / è funesto a chi nasce il dì natale"), è peraltro da segnalare che il lemma è presente anche nei quasi contemporanei versi di "Tempi di Bellosguardo," anche se nella diversa accezione di 'cunetta.' "Ruga," parola descrittiva con cui viene resa l'artificiosa metafora "time's furrows," oltre a recuperare l'antica fonte di Shakespeare, Ovidio ("in speculo rugas adspexit," Met. 15.232), rammenta all'attento lettore uno degli attributi della solita annunciatrice-risvegliatrice della Bufera: "biondo / cinerei i capelli / sulla ruga che tenera / ha abbandonato il cielo" ("Il tuo volo").

Poco da aggiungere sullo "specchio," elemento archetipico, ricorrente nella poesia moderna e segnatamente in Montale (cfr. Avalle 21-33). La rinuncia alla desueta forma "spera" (usata per esempio negli "Orecchini"), rinuncia che ulteriormente indebolisce l'ipotesi che l'immissione di arcaismi fosse finalizzata alla creazione di un linguaggio atto a rendere lo stile shakespeariano, è essenzialmente dovuta alla assonanza di "specchio" con "vecchio." Così, pur avendo sostituito "old" con "giovane," l'incubo della senescenza ritorna fin nel primo verso, inquinando la giovinezza ("ruit hora," un altro grande tema montaliano) e confermando l'ambiguità di ogni cosa umana. In quanto a "beltà," il suo uso in luogo di "bellezza" è probabilmente dovuto a ragioni di ordine ritmico (la sua maggiore concisione) e soprattutto fonetico (l'opportunità di evitare la replica della sgradevole terminazione in "-ezza," già in "Giovinezza”). 
Di sapore indiscutibilmente montaliano è l'immagine della "fida nutrice," da aggiungere all'elenco delle devote serve c dei cani fedeli. Che qui la tematica del ricordo irrompa nel testo e stravolga l'originario significato del sonnel shakespeariano appare evidente: nella "cuna" non viene mostrato alcun bimbo; del "babe" che la "tender nurse" si affanna a conservare in salute non c'è traccia. È l'intera similitudine del v. 12 inglese che si sfalda, diluendosi in due versi semanticamente isolati c sintatticamente incompiuti. L'impressione è piuttosto quella del flash-back, della improvvisa digressione (o anche regressione) verso il passato, provocata da una madeleine, da un keepsake. La frase conclusiva del v. 12, "che ogni morbo stia distante," ha più che altro i caratteri dello scongiuro, della invocazione scaramantica: e diegeticamente va attribuita alla bàlia, che la ripeteva sulla culla del bimbo-poeta.

Assai congruo alla poesia di Montale (ed è stato infatti conservato senza soverchie variazioni, se si eccettua la scomparsa di "breast") è poi il complesso gioco di scambi e sovrapposizioni fra narratore e narratario, di evidentc eredità petrarchista: "del mio . . . nel tuo," "il tuo nel mio," "sul tuo . . . io," "di me: del tuo," "il mio ... il tuo." Si ricordino infatti "tuo nelle mie" ("Ecco il segno; s'innerva"), "del tuo . . . e del mio" ("Incantesimo"), "tra me e te" ("Il fiore che ripete"), "fra te e me" e "con me tu" ("Due nel crepuscolo"), formule che ulteriormente si infittiscono in Xenia e nelle ultime raccolte.

Evidente nel "Sonetto XXII" è poi la polarizzazione del rapporto "io"-"tu" (in inglese indebolito dalla presenza di un pronome di terza persona "her"), amplificata dall'ossessiva iterazione:

Allo specchIO, ancor glOvane MI credo ché GIOvinezza e TE sicTE una cosa.

Ma se una ruga sul TUO volto IO veda saprò che anche per ME morTE non posa.

Quella beltà che TI ravvolge c̀ ancora parvenza del MIO cuore che nel TUO alberga - e il TUO nel MIO-; c coME allora decidere chi è il vecchlO di NOI due?

Poni in scrbo il TUO cuore, ed 10 lo s'TEsso farò di ME: del TUO così zclanTE coME fida nutrice in veglia presso la cuna, che ogni morbo sTla distanTE.

Spento il MIO cuore, invaNO IL, TUO riprendere vorresTI: chi l'ha avuto non lo rende.

È soprattutto significativo ritrovare l" "io" nascosto nelle pieghe dello "specchIO," di "glOvane" e di "GIOvinezza"; e il "tu" in "morTE." Il senso letterale, che conduceva all'equazione "Giovinezza" : "te" = "morte" : "me," risulta rovesciato. E se da un lato questo fatto contribuisce a rivelare il senso più riposto del componimento, dall altro conferma la sfiducia montaliana nelle cose e nelle parole. 
3.4. Il ribaltamento sintattico operato da Montale sull'incipit shakespeariano, per cui autore dello sguardo diventa il poeta, e non più lo specchio personificato, comporta, e il fatto è assai rilevante, l'istituzione di una perfetta simmetria fra la prima e la seconda metà della quartina (fra i cui versi, insolitamente, non sussistono enjambements):

LUOGO: "Allo specchio" vs "sul tuo volto";

TEMPO: "ancor" (=presente, come eredità del passato) vs "se" (=futuro ipotetico); IMPRESSIONE VISIVA: "giovane mi credo" vs "una ruga . . . io veda";

DEDUZIONE RAZIONALE: "ché Giovinezza e te siete una cosa" vs "saprò che anche per me morte non posa."

La prima serie è costituita da elementi semanticamente positivi: "ancor" (=continuazione), "giovane," "Giovinezza," "siete una cosa" (=affermazione di esistenza); l'altra da elementi negativi: "se" (=dubbio), "ruga" (=invecchiamento, perdita della bellezza), "morte," "non posa" (=negazione del riposo). Evidentemente lo "specchio" fornisce un'immagine della realtà più piacevole di quella rivelata dal "volto" dell'amata. Quest'ultima, lungi dall'essere presenza confortante e salvifica, sembra essere pertanto messaggera, se non portatrice, di corruzione e di morte. L'impressione pare confermata nel distico finale: il participio perfetto assoluto "Spento" può venire riconosciuto, all'analisi grammaticale, come predicato di "mio cuore" (soggetto), e dunque come sostitutivo della proposizione riflessiva 'Essendosi spento'; ma alla lettura, soprattutto se non si ha in mente il testo inglese, esso appare piuttosto predicato del "tu" (soggetto sottinteso della frase successiva), con "il mio cuore" complemento oggetto. In questo caso il significato del couplet sarebbe il seguente: 'Dopo aver spento il mio cuore, invano tu vorresti. ...' Oltre che annunciatore di senescenza, il "tu" si rivela responsabile della morte (sia pure figurata) del poeta.

Ancora una volta, però, occorre cautela. Come già verificato nell'analisi del "Sonetto XXXIII," non si deve commettere l'errore di attribuire all'azione del "tu" un valore negativo. L'immagine che si osserva allo specchio è certo attraente, ma purtroppo è falsa, illusoria. Il poeta lo sa: "ancor . . . mi credo," "è ancora / parvenza." "Ancor," "ancora": è il passato che sopravvive oltre se stesso, è l' "io" che cerca di non guardare, che si aggrappa al ricordo di una giovinezza e di una bellezza ormai sfiorite. Il "tu," come sempre fin dai Mottetti, è allora portatore di verità: la ruga sul volto non è solo creduta, ma vista ("io veda"), l'inevitabilità della morte non è solo postulata, ma conosciuta ("saprò"). È un altro sonetto "dell'esperienza," questo, un'altra amara testimonianza della necessità di abbandonare l'età dei sogni e delle illusioni e di guardare in faccia l'aspra tragedia dello stato umano. Ritornano in mente i versi del "Balcone," quell" "imperativo" che apre le Occasioni: "Pareva facile giuoco / mutare in nulla . .."; "Ora . . . / sull'arduo nulla si spunta / l'ansia ..."; "La vita . . . / è quella che sola tu scorgi." E il commento di Fredi Chiappelli: "Passato e futuro non hanno più equipaggio, 
la loro materia cessa, si estingue nel composto del presente in eletti residui incapaci di attuare un'animazione. La figura complessiva è nella percezione di un invanire del vigor dell'età, del fiaccarsi dell'applicazione vitale" (107).

Torniamo al "Sonetto XXII." La domanda del v. 8, "chi è il vecchio di noi due?," la presenza di quel solitario "noi," sanzionano l'avvenuto contatto con l'esperienza, la maturazione. La sentenza conclusiva, "chi l'ha avuto non lo rende," indica l'impossibilità di rifiutare il dono, e di tornare indietro dopo averlo accettato. L'apparizione della "fida nutrice" e della "cuna," lo scongiuro contro la malattia, sono in questa prospettiva un recupero del passato, un passato remoto (quasi un cerchio che si chiude), non più confuso con il presente, ma acceltato quale prodotto dell'azione ristoratrice della memoria.

4. Nei due sonetti esaminati gli elementi connotatori tendono a disporsi in quattro serie (corrispondenti ad altrettante isotopie semantiche), le quali a loro volta possono essere organizzate, mediante un legame di opposizione, in coppie:

\begin{tabular}{|c|c|c|c|c|c|c|}
\hline & FALSITÀ & VS & VERITÀ & INGENUITÀ & VS & ESPERIENZA \\
\hline \multirow[t]{4}{*}{ XXII: } & "specchio" & & "volto" & "giovane" & & "ruga" \\
\hline & "mi credo" & & "veda" & "Giovinezza" & & "morte" \\
\hline & "parvenza" & & "saprò" & "bcltà" & & "vecchio" \\
\hline & & & & "cuna" & & "nutrice" \\
\hline \multirow[t]{5}{*}{ XXXIII: } & "lusingar" & & "intorbidata" & $\begin{array}{l}\text { "occhio del } \\
\text { mattino" }\end{array}$ & & "fumi" \\
\hline & $\begin{array}{l}\text { "baciar } \\
\text { d'oro" }\end{array}$ & & "fuggendo" & "oro" & & "vili" \\
\hline & $\begin{array}{c}\text { "accendere } \\
\text { pallidi" }\end{array}$ & & "celare" & $\begin{array}{l}\text { "celestiale } \\
\text { fronte" }\end{array}$ & & $\begin{array}{l}\text { "desolato } \\
\text { mondo" }\end{array}$ \\
\hline & "alchimie" & & "onta" & "sole" & & "nubi" \\
\hline & & & & "trionfo" & & "ahimè" \\
\hline
\end{tabular}

Le opposizioni, benché inconciliabili, non sono manichee, cioè non implicano un giudizio di valore: l'ingenuità, anche se spesso confortante e piacevole, non è il bene; l'esperienza, pur dolorosa, non è il male. Un principio di necessità, una sorta di "provvidenza" della natura, governa l'intero meccanismo, e tutti i poli sono importanti e ineliminabili: la verità presuppone la falsità per avere un senso, la conoscenza non può che fondarsi su una tabula rasa, sull'ignoranza. Il processo è in ogni caso irreversibile, e il segno di ciò è lo scorrere del tempo (l'abbuiarsi del sole nel "Sonetto XXXIII," l'invecchiamento nel "Sonetto XXII").

Al punto d'incontro fra l'ordito del tempo e la trama della necessità, all'uomo non resta che la capacità di non sdegnarsi ("Son. XXXIII," v. 13), la volontà di non rinnegare la conquistata (e bruciante) maturità ("Son. XXII," v. 14). Né c'è premio alcuno, presente o futuro, per questa dignitosa accet- 
tazione delle cose; neppure la speranza di un riconoscimento. Veramente la "tollerabilità del vivere" è a un minimum. Il fatto è che il momento dell'esperienza, che in Montale scattò visibilmente intorno al 1936, quando il poeta entrò nel mezzo del cammin di sua vita, è anche il momento in cui inizia, o comunque si rafforza, l'horror vacui. La poesia della Bufera e dei nostri sonetti (e in prospettiva la produzione del Montale anziano) nasce di qui: dal bisogno di contrastare, ma prima di tutto di accettare, il niente che è al fondo dell'angoscia che stringe la cultura occidentale (cfr. Greco 172-73). Contro il terrore della morte, Montale non poté, o piuttosto non volle, aggrapparsi alla fede o al desiderio di una vita ultraterrena, e neppure ricorrere a un'ancor più problematica fiducia nelle magnifiche sorti e progressive. Unico rimedio all'impasse fu per lui allora una lucida, personale (ancorché esemplare) "forza segreta di stoico" (Scrivano 305).

Strumenti gnoseologici, e non consolazione, Montale ha cercato e trovato nell'opera dei suoi "padri metafisici," nella fattispecie in Shakespeare. Lo stesso dobbiamo noi cercare e trovare nella sua lirica. L'amore e soprattutto la morte sono gli oggetti della sua indagine; il fascismo, la guerra, il dopoguerra, sono temi di importanza assolutamente secondaria, semplice sfondo a una coraggiosa e faticosa (ma forse anche inevitabile) scelta di verità. Non casuali allora, ma autentico segnale dell'inizio di una nuova (e definitiva) fase poetica sono i due versi posti in epigrafe all'ultima sezione delle Occasioni, tratti da un componimento (Sonnets 5) in cui Shakespeare celebra la possibilità dei genitori di sopravvivere nei figli, ma che di quel componimento costituiscono il momento più negativo, l'amara metafora della decadenza e della fine:

Sap check'd with frost, and lusty leaves quite gone, Beauty o'ersnow'd and bareness every where.

\section{University of Toronto}

\section{NOTES}

1 Dei numerosi saggi su Montale traduttore - oltre a quelli specificamente citati nel corso della mia analisi, c dunque compresi nella bibliografia - sono da ricordare almeno: Marisa Bulgheroni, "Dickinson/Montale: il passo sull'erba," Eugenio Montale, a cura di Annalisa Cima c Cesare Segre (Milano: Rizzoli, 1977) 91-114; c Pier Vincenzo Mengaldo, "La panchina e i morti (su una versione di Montale)," La tradizione del Novecento: mova serie. Firenze: Vallccchi, 1987. 215-34. Mentre questo saggio è in bozze, escono il Quaderno momaliano, a cura di Pier Vincenzo Mengaldo (Padova: Liviana, 1989), nel quale a Montale traduttore (di Yeats) sono dedicati tre interventi; e il bel libro di Laura Barile, Adorate mie larve. Montale e la poesia anglosassone (Bologna: II Mulino, 1990).

2 Cfr. la "Nota dei curatori" in Montale, L'opera in versi 829-40. Da questa edizione cito tutte le poesic montaliane, comprese le versioni dei Sonnets (queste ultime sono a pp. 711-13). I icsti shakespeariani sono invece quelli che appaiono, a fronte delle rispettive traduzioni, nel Quaderno di traduzioni (Milano: Mondadori, 1975) 16-18.

3 Qui da pronunciarc/alki'mai/ per effetto della rima obbligata col precedentc "eye."

4 Cfr. Giorgio Barbcri Squarotti, "La sloria," Letture Montaliane 281-96, e Scrivano.

5 Ed è un caso che nell'ultimo Montale, con diverso significato, ricompaia però la medesima immagine, riferita a un altro dei grandissimi temi del nostro poeta, la memoria? "Si aggiungc all'csistente come un'aureola / di nebbia al capo ..." ("La memoria"). 
6 E vedi anche la terza strofe, poi soppressa, delle prime cuizioni di "Vasca"; in cui l'ora non è quella dell'alba, ma il passare delle nubi d̀ ancora un 'immagine che rimanda esplicitamente allo scorrere del tempo: "Ancora nell' ingannevole ancllo / trapassano le carovane nell'aria, / e meglio vi si stemprano allora quando snello / il fugace zampillo in alto svaria. / Vanno c non lasciano segno / in codesto concluso mondo / anche le nostre giornate" (/ "opera in versi 882). A proposito di "Fine dell"infanzia," non mi pare convineente la riserva di Ettore Bonora, per il quale proprio in quei versi "l'idea delle nuvole ha meno fascino poctico, per il prevaricare del pensiero sulla fantasia" (129). Il paragone con "Corno inglese" o con "Casa sul mare" non costituisce un convineente sistema di giudizio: perché lì l'immagine era sussidiaria, qui costituisce la struttura significante di tutta la seconda parte di un componimento fra i più lunghi dell'intera produzione montaliana. Ciò che in "Fine dell'infanzia" importava al pocta cra istituire una precisa corrispondenza fra il passare delle nuvole e lo scorrere del tempo (analogamente a quanto avrebbe anni dopo fatto avvicinando al tempo l'acqua dei fiumi, in una delle grandi poesic di Satura, "L'Arno a Rovezzano"). Un secondo grado di metaforizzazione avrebbe finito col risultare inutile $\mathrm{e}$ forse faticoso.

\section{BIBLIOGRAFIA}

Avallc, D'Arco Silvio. Tre saggi su Montale. Torino: Einaudi, 1970.

Barberi Squarotti, Giorgio. "La metrica e altro." Gli inferi e il labirinto: da Pascoli a Montale. Bologna: Cappelli, 1974. 195-209.

Benjamin, Walter. Angelus Norus: saggi e frammenti. Trad. ital. di Renato Solmi. Torino: Einaudi, 1962.

Bigongiari, Picro. “Altri dati per la storia di Montalc." Poesia italiana del Novecento. Firenze: Vallecchi, 1965. 226-41.

Bonora, Ettore. Letura di Montale. I: Ossi di seppia. Torino: Tirrenia, 1980.

Carpi, Umberto. Montale dopo il fascismo dalla Bufera a Satura. Padova: Liviana, 1971.

Chiappelli, Fredi. "L'imperativo e l'occasione." Letture Montaliane 105-10.

Greco, Lorenzo. Montale commenta Montale. Parma: Pratiche, 1980.

Leture Montaliane in occasione dell' 80 o complcanno del Poeta. Genova: Bozzi, 1977.

Lonardi, Gilberto. "Fuori c dentro il tradurre montaliano." Il Vecchio e il Giosane e altri studi su Montale. Bologna: Zanichelli, 1980. 144-63.

Mcoli Toulmin, Rachel. "Shakespeare ed Eliot nelle versioni di Eugenio Montale." Belfagor 26 (1971): 435-71.

Montalc, Eugenio, trad. Amleto principe di Danimarca. Di William Shakespeare. Milano: Cederna, 1949.

. Auto da fé: cronache in due tempi. Milano: II Saggiatore, 1966.

Intervista. Il mestiere di poeta. Di Ferdinando Camon. Milano: Garzanti, 1982.

$23-28$.

Einaudi, 1980.

L'opera in iersi. A cura di Rosanna Bettarini e Gianfranco Contini. Torino: Sulla poesia. A cura di Giorgio Zampa. Milano: Mondadori, 1976.

Musatti, Maria Pia. "Montale traduttore: la mediazione della pocsia." Strumenti critici 14 (1980): 122-48.

Scrivano, Riccardo. "La storia." Leture Montaliane 297-320.

Serpieri, Alessandro. I sonetti dell immortalita: il problema dell arte e della nominazione in Shakespeare. 2a cd. Milano: Bompiani, 1976.

Singh, Ghanshyam. "Montale e la poesia inglese." La poesia di Eugenio Vontale: Autu del Conegno Internazionale tenuto a Genova dal 25 al 28 notcmbre 19,82. A cura di Surgio Campailla e Cesare Federico Goffis. Firenze: Le Monnier, 1984. 205-20.

Ungaretti, Giuseppe, trad. 40 sonetli di Shakespearc. Milano: Mondadori, 1946. 